\title{
Two-Phase Thermal Switching System for a Small, Extended Duration Lunar Surface Science Platform
}

\author{
D. C. Bugby ${ }^{\mathrm{a}}$, J. T. Farmer ${ }^{\mathrm{b}}$, B. F. O’ Connor $^{\mathrm{c}}$, \\ M. J. Wirzburger ${ }^{d}$, E. D. Abel ${ }^{\mathrm{e}}$, and C. J. Stouffer ${ }^{\mathrm{f}}$ \\ ${ }^{a}$ ATK Space, 5050 Powder Mill Road, Beltsville, MD 20705 \\ (301) 902-4385;david.bugby@atk.com \\ ${ }^{b}$ NASA Marshall Space Flight Center, MFSC, Huntsville, AL 35812 \\ (256) 544-3179; jeffery.t.farmer@nasa.gov \\ ${ }^{c}$ NASA Marshall Space Flight Center, MFSC, Huntsville, AL 35812 \\ (256) 544-6649; brian.f.oconnor@nasa.gov \\ ${ }^{d}$ Johns Hopkins University Applied Physics Laboratory, 11100 Johns Hopkins Road, Laurel, MD 20723 \\ (240) 228-5584; melissa.wirzburger@jhuapl.edu \\ ${ }^{e}$ Johns Hopkins University Applied Physics Laboratory, 11100 Johns Hopkins Road, Laurel, MD 20723 \\ (240) 228-7704; elisabeth.abel@jhuapl.edu \\ ${ }^{f}$ ATK Space, 5050 Powder Mill Road, Beltsville, MD 20705 \\ (301) 902-4404;chuck.stouffer@atk.com
}

\begin{abstract}
This paper describes a novel thermal control system for the Warm Electronics Box (WEB) on board a small lunar surface lander intended to support science activities anywhere on the lunar surface for an extended duration of up to 6 years. Virtually all lander electronics, which collectively dissipate about $60 \mathrm{~W}$ in the reference mission, are contained within the WEB. These devices must be maintained below $323 \mathrm{~K}$ (with a goal of $303 \mathrm{~K}$ ) during the nearly 15-earth-day lunar day, when surface temperatures can reach $390 \mathrm{~K}$, and above $263 \mathrm{~K}$ during the nearly 15-earth-day lunar night, when surface temperatures can reach $100 \mathrm{~K}$. Because of the large temperature swing from lunar day-to-night, a novel thermal switching system was required that would be able to provide high conductance from WEB to radiator(s) during the hot lunar day and low (or negligible) conductance during the cold lunar night. The concept that was developed consists of ammonia variable conductance heat pipes (VCHPs) to collect heat from WEB components and a polymer wick propylene loop heat pipe (LHP) to transport the collected heat to the radiator(s). The VCHPs autonomously maximize transport when the WEB is warm and autonomously shut down when the WEB gets cold. The LHP autonomously shuts down when the VCHPs shut down. When the environment transitions from lunar night to day, the VCHPs and LHP autonomously turn back on. Out of 26 analyzed systems, this novel arrangement was able to best achieve the combined goals of zero control power, autonomous operation, long life, low complexity, low $\Delta \mathrm{T}$, and landed tilt tolerance.
\end{abstract}

Keywords: lunar lander, spacecraft, thermal control, thermal switch, two-phase, heat transfer, variable conductance, heat pipe PACS: 07.20.Pe, 07.87.+v, 44.00.00, 44.10+i, 44.30.+v, 44.35.+c, 68.03.Cd, 68.03.Fg, 94.05.Hk, 96.25.Vt

\section{INTRODUCTION}

Extended life lunar surface science platforms, using solar/battery or radioisotope power, require advanced thermal switching systems that consume minimal electrical heater power during the cryogenically cold 15-earth-day lunar night and provide highly efficient cooling during the searingly hot 15-earth-day lunar day. This paper describes an analytical study of thermal switching systems that can best meet the thermal requirements of electronics/batteries on a range of lunar missions including the International Lunar Network (ILN) anchor node spacecraft. 
The ILN program seeks to establish a network of lunar geophysical science platforms in order to better understand the interior structure and composition of the moon. This surface science capability has been long sought-after by the lunar science community ever since the Apollo seismic stations ceased operation in 1977. ILN anchor node spacecraft are small, low-power, long-life (6-year) landers with seismographic and a few other science instruments. Figure 1 illustrates notional sketches of anchor node spacecraft based on the information available in mid-summer 2009 (concepts may have changed slightly since then). As indicated, nearly all anchor node electronics/batteries are contained within an enclosure known as the Warm Electronics Box (WEB). Defining the optimum thermal control system to keep the WEB warm during the lunar night and cool during the lunar day was the technical problem addressed herein. The remainder of the paper describes the trade study performed to address that problem and it is organized into the following remaining sections: problem, concept, analysis, plans, and conclusion.
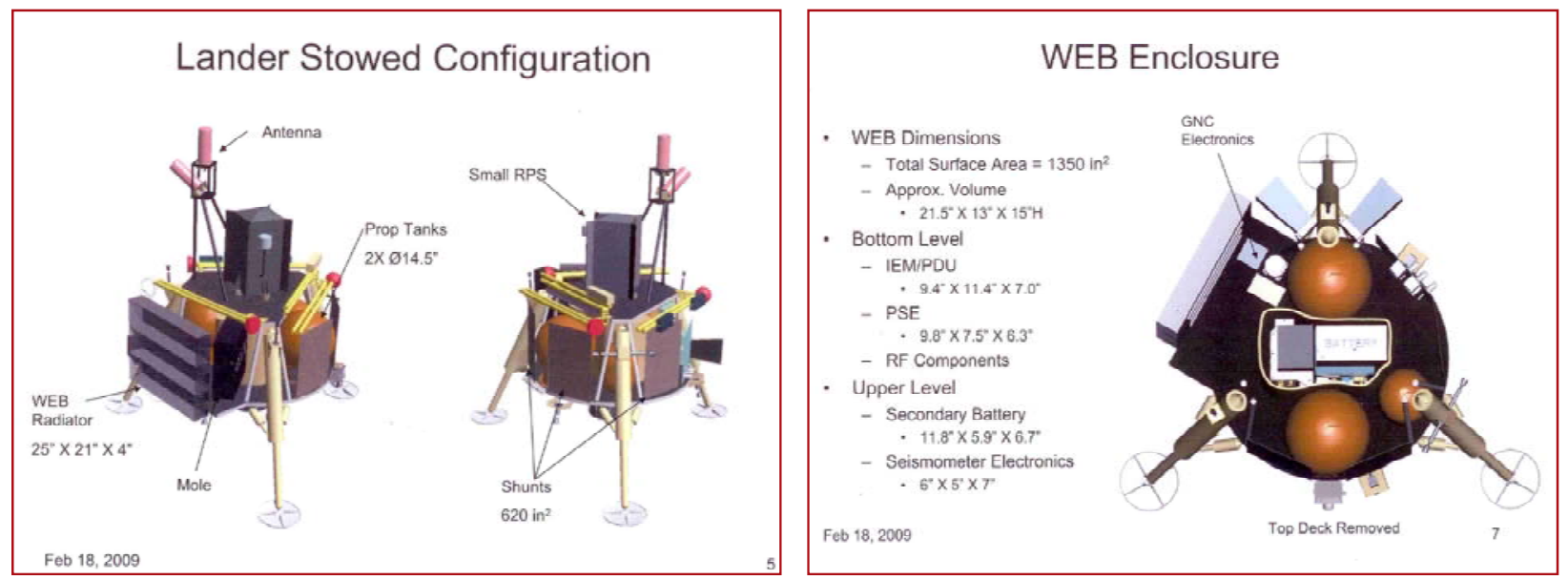

FIGURE 1. ILN Anchor Node Spacecraft (Based on Mid-Summer 2009 Information)

\section{PROBLEM}

To provide the requisite thermal control, the fundamental technical need is a variable conductance heat transport system (VCHTS) that links the WEB to one or more radiators. A simple block diagram of the VCHTS, WEB, and a single radiator is provided in Figure 2. A more realistic system diagram, which includes the possibility of multiple intra-system interfaces, including a segmented radiator, is shown in Figure 3. Using Figure 3 as the starting point, the WEB-to-radiator heat transport path can be broken down as indicated in Figure 4, which depicts potential subsystems, interfaces, and system options (note: Figure 4 is color-coded to match Figure 3). The system options are distinct heat transport path arrangements that offer varying degrees of "vertical integration (VI)", where the degree of VI increases as the number of heat transport path interfaces decreases. Therefore, minimum VI, which is characterized by the maximum number of subsystem interfaces and the largest system $\Delta \mathrm{T}$, is where the VCHTS thermally links the WEB to the radiator but does not isothermalize the WEB, which can contain multiple electronics boxes/batteries, and does not isothermalize the radiator, which may be bladed as shown in Figure 1. Full VI, which is characterized by the minimum number of subsystem interfaces and the smallest system $\Delta \mathrm{T}$, is where the VCHTS isothermalizes the WEB, provides the requisite thermal transport/switching, and also isothermalizes the radiator.

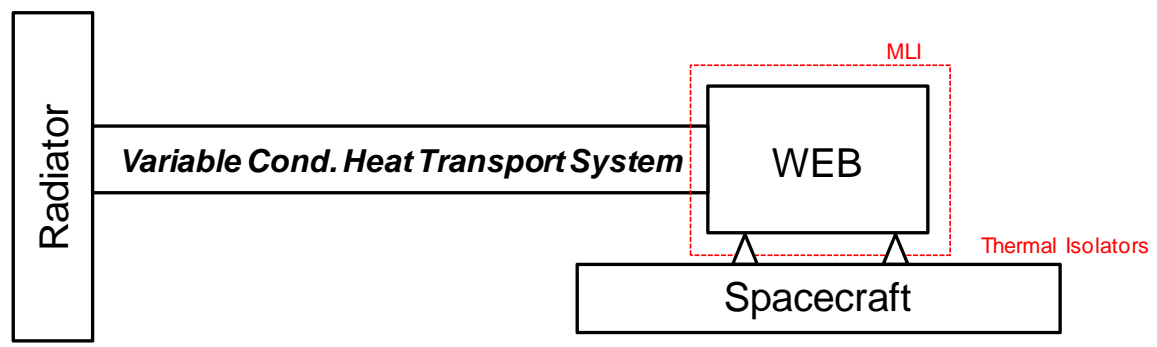

FIGURE 2. Simple System Diagram 


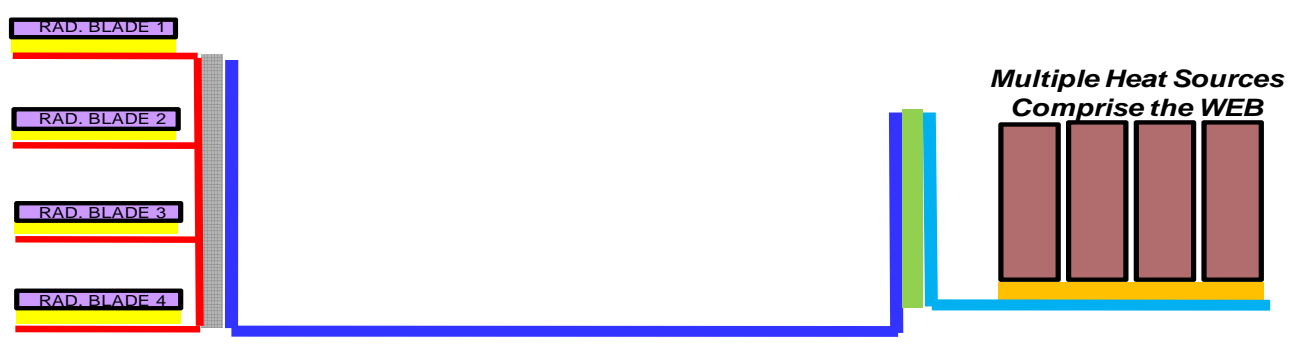

FIGURE 3. Realistic System Diagram

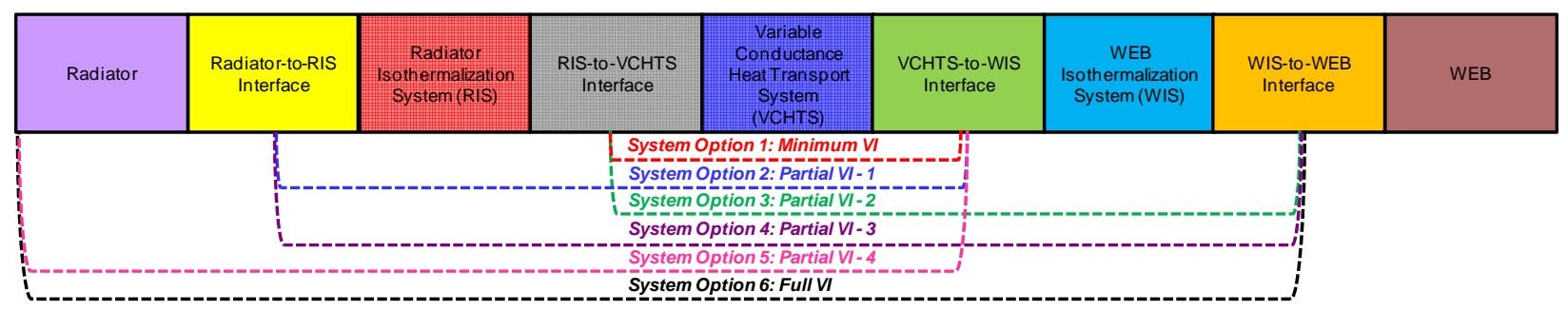

FIGURE 4. Heat Transport Path: Subsystems, Interfaces, and System Options

With the foregoing in mind, the key thermal design targets that were stated by NASA (or were inferred by the analysis team) are as follows: (1) WEB operating temperature (263-323 K, with 263-303 K as a goal); (2) duration of lunar day/night (about 15 earth-days each); (3) lunar day sink temperature (260 K with an appropriately oriented radiator that runs much colder than the $390 \mathrm{~K}$ lunar day surface temperature); (4) lunar night sink temperature (60-150 K, depending on the modeling assumptions, which were not fully defined at the time of this study); (5) cruise sink temperature (TBD, at the time of this study); (6) cruise maximum spin rate (6 rpm, which produces a small $0.03 \mathrm{~g}$ acceleration with a $0.75 \mathrm{~m}$ moment arm); (7) WEB power during lunar day (60 W); (8) WEB power during lunar night (20 W for solar/battery power, $60 \mathrm{~W}$ for radioisotope power); (9) transported power during lunar night ( $0 \mathrm{~W}$ for solar/battery, $40-55 \mathrm{~W}$ for radioisotope power); (10) radiator emissivity (0.93 nominal, 0.70 degraded); (11) radiator area $\left(0.26-0.34 \mathrm{~m}^{2}\right.$; current designs may have multiple, larger radiators); (12) radiator temperature during lunar day (298 K with $60 \mathrm{~W}, 0.93$ emissivity, and $0.34 \mathrm{~m}^{2}$ radiator area; current designs may have multiple, larger radiators); (13) minimum ON conductance (6 W/K; $12 \mathrm{~W} / \mathrm{K}$ goal); (14) radiator temperature during lunar night (same as sink temperature, with $0 \mathrm{~W} / \mathrm{K}$ OFF conductance); (15) OFF conductance (adjustable down to nearly $0 \mathrm{~W} / \mathrm{K}$ based on WEB temperature); (16) ON/OFF conductance ratio (maximize); (17) ON-to-OFF switching time (minimize); (18) start-up (minimize need for special procedures; goal is autonomous); (19) control power (minimize; $5.5 \mathrm{~kg}$ mass penalty per $1 \mathrm{~W}$ of control power); (20) mass (minimize); (21) volume (minimize); (22) cost (minimize; but need to define the required technology development); (23) lifetime (6 years minimum); (24) transport length (0.5-1.75 m; NASA estimate); (25) flight heritage (desired; not required); (26) vertical integration (VI) capability (maximize; assume WEB interface cannot be eliminated); (27) complexity (minimize; use simplest system possible); (28) autonomous operation (desired; not required); (29) scalability (maximize; should be able to accommodate increases/decreases in power); (30) testability (should be testable in 1g); (31) test orientation (flight units to be ground-testable in flat orientation); (32) tilt tolerance (to the potential landed adverse tilt of $+/-20^{\circ}$ ); (33) ease of integration (maximize; should be easy to integrate); (34) environment compatibility (maximize; should be operable in lunar environment); and (35) redundancy (not required).

To reduce the 35 requirements listed above into a more manageable number for concept development and trade study evaluation, six primary system discriminators were identified based on the judgment of the analysis team after introductory project discussions with NASA. Those six primary system discriminators, which are identified (above) in red type, include (19) control power, (23) lifetime, (26) vertical integration (VI) capability, (27) complexity, (28) autonomous operation, and (32) landed adverse tilt. The approach utilized in the trades that follow was to define several potential thermal control system concepts, assign a score to each concept in each of the six system discriminator categories, combine the system discriminator scores into a total concept score, and then rank the concepts based on the total score. The next section will describe the various concepts that were considered. 


\section{CONCEPT}

Five generic two-phase thermal transport/switching concepts were considered in this trade study. Those five concepts - variable conductance heat pipe (VCHP), capillary pumped loop (CPL), loop heat pipe (LHP), hybrid loop heat pipe (HLHP), and mechanically pumped two-phase loop (MPTPL) - are listed in Table 1 along with an assessment of their ground/flight heritage. To enhance the performance of each system (i.e., reduce control power, provide better switching, and/or increase conductance), seven potential system modifications were considered in this trade study. Those seven potential modifications - thermoelectric cooler (TEC), flow-obstructing valve (FV), bypass valve (BV), heat exchanger plus subcooler ( $H X+S C)$, VCHP, multiple evaporator/condenser (MEC), and multi-pass system (MP) - are listed in Table 2 along with their function and ground/flight heritage. Figure 5 illustrates the five generic thermal switching systems (note: "heat in" corresponds to heat input from the WEB, "heat out" corresponds to heat rejected to the radiator). Figure 6 depicts the seven system enhancers (clockwise beginning at the lower left).

Table 1. Generic Thermal Transport/Switching Systems Considered

\begin{tabular}{llll}
\hline \multicolumn{1}{c}{ System } & \multicolumn{1}{c}{ Definition } & Thermal Switching Mechanism, Actuator & Heritage \\
\hline \hline 1. VCHP & Variable Conductance Heat Pipe & NCG condenser blockage, reservoir heater & Flight (Extensive) \\
\hline 2. CPL & Capillary Pumped Loop & Liquid condenser blockage, reservoir heater & Flight (Moderate) \\
\hline 3. LHP & Loop Heat Pipe & Liquid condenser blockage, reservoir heater & Flight (Extensive) \\
\hline 4. HLHP & Hybrid Loop Heat Pipe & Liquid condenser blockage, reservoir heater & Ground (Extensive) \\
\hline 5. MPTPL & Mechanically Pumped Two-Phase Loop & Liquid flow rate, pump power (adjusts speed) & Ground (Moderate) \\
\hline
\end{tabular}

Table 2. Modifications to Enhance Generic System Performance

\begin{tabular}{lll}
\hline \multicolumn{1}{c}{ System Modification } & \multicolumn{1}{c}{ Performance Enhancement Mechanism } & Heritage \\
\hline \hline A. Thermoelectric Cooler (TEC) & Reservoir cooling for robustness, lower control power & Ground \\
\hline B. Flow-Obstructing Valve (FV) & Actuating valve to block flow for ON/OFF functionality & Ground \\
\hline C. Bypass Valve (BV) & Pressure actuated valve for LHP ON/OFF functionality & Ground \\
\hline D. HX + Subcooler (HX+SC) & Modification to LHP to reduce control power & Ground \\
\hline E. VCHP & Thermal switching/transport/isothermalization (addition) & Flight \\
\hline F. Multiple Evaporator/Condenser (MEC) & Multiple evaporator/condenser for higher conductance & Flight \\
\hline G. Multi-Pass System (MP) & Multi-pass flow routing for higher conductance & Concept \\
\hline
\end{tabular}

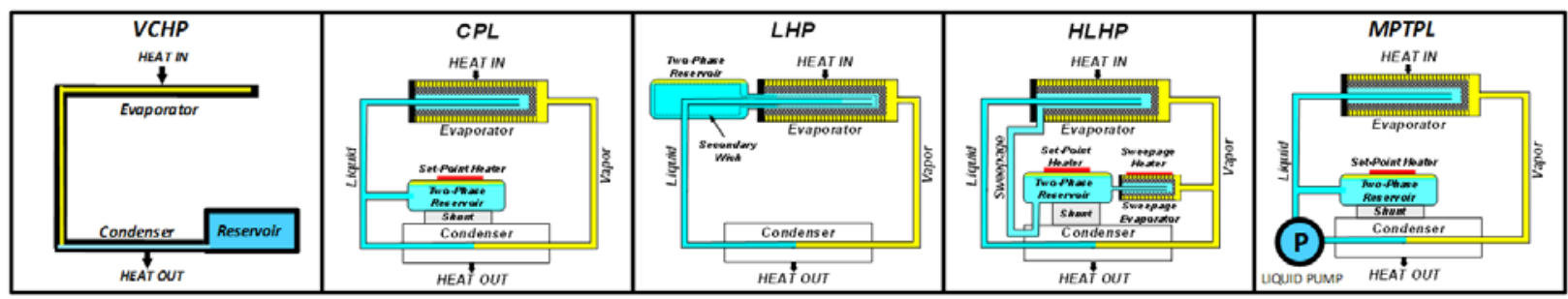

FIGURE 5. Generic Two-Phase Thermal/Transport Switching Systems Considered

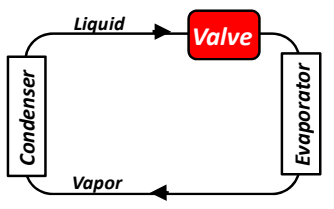

B. FV

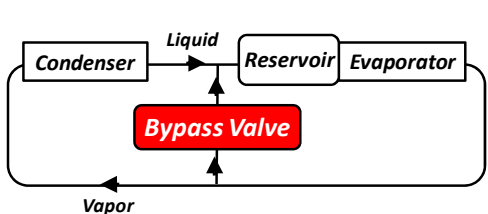

C. BV

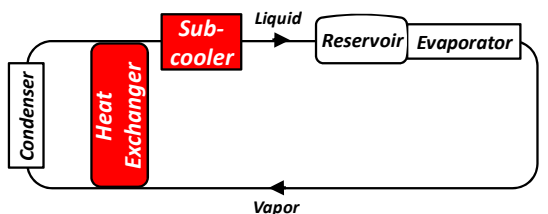

D. $H X+S C$



A. TEC

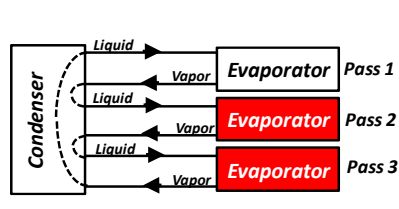

G. MP

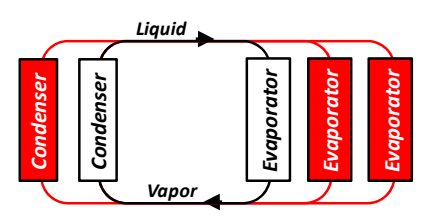

F. MEC

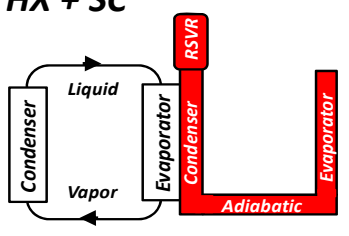

E. VCHP

FIGURE 6. System Modifications to Enhance Generic System Performance 


\section{ANALYIS}

By appropriately combining the five aforementioned thermal transport/switching system options and seven potential performance enhancements, a total of 26 systems were analyzed. The analyzed systems included the following: (1) VCHP; (2) CPL; (3) LHP; (4) HLHP; (5) MPTPL; (6-8) CPL, LHP ,HLHP with TEC; (9-11) CPL, LHP, HLHP with FV; (12) LHP with BV; (13) LHP with HX+SC; (14-17) VCHP, CPL, LHP, HLHP with VCHP; (18-20) CPL, LHP, HLHP with MP; (21-23) CPL, LHP, HLHP with MP and VCHP; (24-26) CPL, LHP, HLHP with MEC. The analytical process used to rank the systems was to assign each system a score from 0-4 (using increments of 0.5) in each of the six system discriminator categories (i.e., control power, lifetime, capability for VI, complexity, autonomous operation, and tilt tolerance). Scoring was carried out using the Table 3 scoring methodology template (note: scores were generated based on a subjective assessment by the analysis team and Table 3 was actually used to "explain" the scores generated). To ensure the selected system would perform well in all six categories, the total score $\left(\mathrm{TS}_{\mathrm{i}}\right)$ for each system (i) was defined as the product of the scores $\left(\mathrm{S}_{\mathrm{i}, \mathrm{j}}\right)$ in each discriminator category $(\mathrm{j})$. Equation (1) indicates the total scoring function, the results of which are provided in Table 4.

$$
\mathrm{TS}_{\mathrm{i}}=\mathrm{S}_{\mathrm{i}, 1} * \mathrm{~S}_{\mathrm{i}, 2} * \mathrm{~S}_{\mathrm{i}, 3} * \mathrm{~S}_{\mathrm{i}, 4} * \mathrm{~S}_{\mathrm{i}, 5} * \mathrm{~S}_{\mathrm{i}, 6}
$$

Table 3. System Discriminator Scoring Methodology

\begin{tabular}{|c|c|c|c|c|c|c|c|c|c|c|}
\hline \multirow{2}{*}{ Category } & \multirow{2}{*}{ Units } & \multirow{2}{*}{$\begin{array}{c}\text { Worst } \\
0.0 \\
\end{array}$} & \multicolumn{6}{|c|}{ Score } & \multicolumn{2}{|r|}{ Best } \\
\hline & & & 0.5 & 1.0 & 1.5 & 2.0 & 2.5 & 3.0 & 3.5 & 4.0 \\
\hline Control Power & $\mathrm{WW}$ & $5+$ & $4-5$ & $3-4$ & $2-3$ & $1-2$ & $3 / 4-1$ & $1 / 2-3 / 4$ & $<<1 / 4$ & 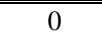 \\
\hline Lifetime & Yrs & $0-2$ & $2-3$ & $3-4$ & $4-5$ & $5-6$ & $6-7$ & $7-8$ & $8-10$ & $10+$ \\
\hline Capability for $\mathrm{VI}^{1}$ & & $n p^{2}$ & $<\min \mathrm{VI}$ & $\min \mathrm{VI}$ & pVI-1 & pVI-2 & pVI-3 & pVI-4 & pVI-5 & full VI \\
\hline Complexity & & np & high & & & medium & & & & low \\
\hline Autonomous Oper. ${ }^{3}$ & & np & & smart & & & dumb & & & auto \\
\hline Tilt Tolerance & & $\mathrm{np}$ & $\min$ & $1.5 \mathrm{X} \min$ & $2 \mathrm{X} \min$ & $2.5 \mathrm{X} \min$ & $3 X \min$ & $3.5 \mathrm{X} \min$ & $4 \mathrm{X} \min$ & $5 \mathrm{X}^{+} \min$ \\
\hline
\end{tabular}

${ }^{1} \mathrm{p}$ VI = partial VI (see Figure 4); ${ }^{2} \mathrm{np}=$ not possible; ${ }^{3}$ smart $=$ microprocessor, dumb = mechanical thermostat, auto $=$ autonomous

Table 4. System Scoring Results

\begin{tabular}{|c|c|c|c|c|c|c|c|c|}
\hline System & Control Power & Lifetime & VI Ability & Complexity & Autonomy & Tilt Tolerance & Score & Conclusion \\
\hline 1. VCHP & 4.0 & 4.0 & 2.0 & 4.0 & 4.0 & 1.0 & 512 & 4 \\
\hline 2. $\mathrm{CPL}$ & 1.0 & 4.0 & 3.0 & 2.5 & 1.0 & 4.0 & 120 & Eliminate \\
\hline 3. LHP & 1.0 & 4.0 & 3.0 & 3.0 & 2.0 & 4.0 & 288 & Eliminate \\
\hline 4. HLHP & 1.0 & 4.0 & 3.0 & 2.0 & 1.0 & 4.0 & 96 & Eliminate \\
\hline 5. MPTPL & 2.0 & 1.0 & 3.0 & 2.5 & 3.0 & 4.0 & 180 & Eliminate \\
\hline 6. CPL/TEC & 2.0 & 2.0 & 3.0 & 2.0 & 1.0 & 4.0 & 96 & Eliminate \\
\hline 7. LHP/TEC & 2.0 & 2.0 & 3.0 & 2.5 & 1.5 & 4.0 & 180 & Eliminate \\
\hline 8. HLHP/TEC & 2.0 & 2.0 & 3.0 & 1.5 & 1.0 & 4.0 & 72 & Eliminate \\
\hline 9. CPL/FV & 1.0 & 1.0 & 3.0 & 1.5 & 1.0 & 4.0 & 18 & Eliminate \\
\hline 10. LHP/FV & 1.0 & 1.0 & 3.0 & 2.0 & 1.0 & 4.0 & 24 & Eliminate \\
\hline 11. HLHP/FV & 1.0 & 1.0 & 3.0 & 1.0 & 1.0 & 4.0 & 12 & Eliminate \\
\hline 12. LHP/BV & 4.0 & 1.0 & 3.0 & 2.5 & 4.0 & 4.0 & 480 & 6 \\
\hline 13. $\mathrm{LHP} / \mathrm{HX}+\mathrm{SC}$ & 3.5 & 4.0 & 3.0 & 2.0 & 2.0 & 4.0 & 672 & 3 \\
\hline 14. VCHP/VCHP & 4.0 & 4.0 & 2.5 & 3.5 & 4.0 & 2.0 & 1120 & 2 \\
\hline 15. CPL/VCHP & 1.0 & 4.0 & 4.0 & 2.0 & 2.0 & 4.0 & 256 & Eliminate \\
\hline 16. LHP/VCHP & 3.5 & 4.0 & 4.0 & 2.5 & 4.0 & 4.0 & 2240 & 1 \\
\hline 17. HLHP/VCHP & 1.0 & 4.0 & 4.0 & 1.5 & 1.0 & 4.0 & 96 & Eliminate \\
\hline 18. CPL/MP & 0.5 & 4.0 & 4.0 & 1.0 & 1.0 & 4.0 & 32 & Eliminate \\
\hline 19. LHP/MP & 0.5 & 4.0 & 4.0 & 1.0 & 2.0 & 4.0 & 64 & Eliminate \\
\hline 20. HLHP/MP & 0.5 & 4.0 & 4.0 & 1.0 & 1.0 & 4.0 & 32 & Eliminate \\
\hline 21. CPL/VCHP/MP & 1.0 & 4.0 & 4.0 & 0.5 & 1.0 & 4.0 & 32 & Eliminate \\
\hline 22. LHP/VCHP/MP & 3.5 & 4.0 & 4.0 & 0.5 & 4.0 & 4.0 & 448 & 5 \\
\hline 23. HLHP/VCHP/MP & 1.0 & 4.0 & 4.0 & 0.5 & 1.0 & 4.0 & 32 & Eliminate \\
\hline 24. CPL/MEC & 1.0 & 4.0 & 4.0 & 1.0 & 1.0 & 4.0 & 64 & Eliminate \\
\hline 25. LHP/MEC & 1.0 & 4.0 & 4.0 & 1.0 & 2.0 & 4.0 & 128 & Eliminate \\
\hline 26. HLHP/MEC & 1.0 & 4.0 & 4.0 & 1.0 & 1.0 & 4.0 & 64 & Eliminate \\
\hline
\end{tabular}


The results in Table 4 indicate that the two top-ranked systems are the LHP/VCHP (16) and the VCHP/VCHP (14). Four other highly ranked systems, considered potential backup options, included the LHP/HX+SC (13), VCHP (1), LHP/VCHP/MP (22), and LHP/BV (12). All other systems were eliminated from consideration. Conceptual layouts of the LHP/VCHP and the VCHP/VCHP options are provided in Figure 7 (note: although shown separated, the LHP evaporator in the LHP/VCHP system mates to the two VCHP condensers while the VCHP-R evaporator in the VCHP/VCHP system mates to the two VCHP-W condensers; R and W refer to the radiator and WEB, respectively).
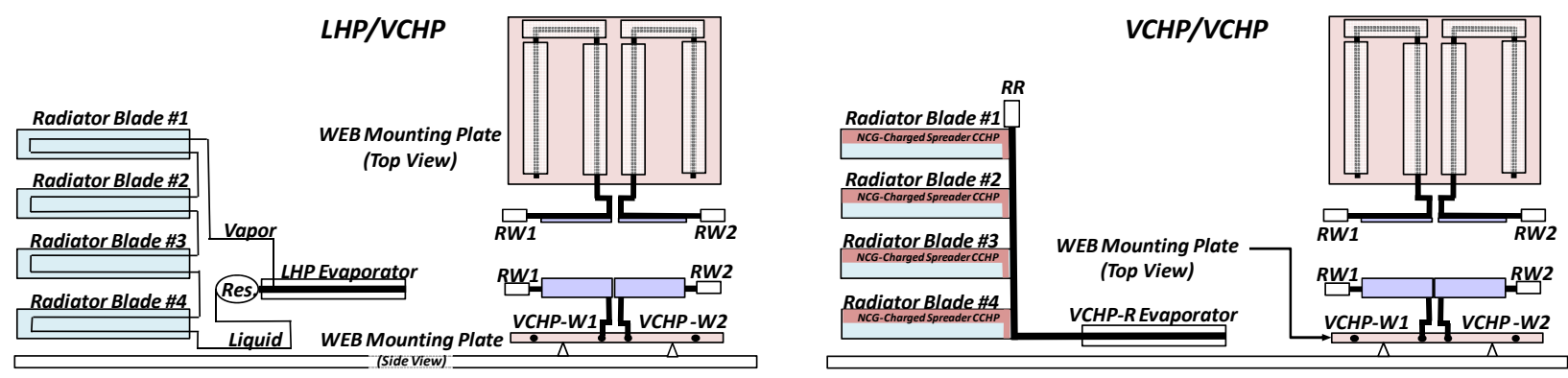

FIGURE 7. Conceptual Layouts of LHP/VCHP and VCHP/VCHP

The LHP/VCHP system utilizes a propylene LHP heat transport subsystem to avoid working fluid freezing in the radiator lines (propylene freezes at $90 \mathrm{~K}$ ) and two ammonia VCHPs to provide WEB isothermalization/switching. The VCHP reservoirs are sized to autonomously block the condenser/adiabatic sections (with NCG) when the WEB gets cold and to autonomously unblock those sections when the WEB warms. The polymer wick LHP autonomously starts up at low power as the VCHP condenser opens upon WEB warming and autonomously shuts down as the VCHP condenser closes upon WEB cooling. The LHP evaporator will be contained within an MLI blanket that surrounds the WEB, thus a small radiative load on the LHP reservoir will prevent it from continuing to cool as the environment cools. The LHP/VCHP system is thus an autonomous thermal transport/switching system.

The VCHP/VCHP system utilizes an ammonia VCHP-R heat transport subsystem, NCG-charged CCHP radiator heat spreaders, and two ammonia VCHP-Ws to provide WEB isothermalization/switching. The VCHP-W reservoirs are sized to autonomously block the condenser/adiabatic sections (with NCG) when the WEB gets cold and to autonomously unblock those sections as the WEB warms. The VCHP-R autonomously starts up at low power as the VCHP-W condenser opens upon WEB warming and autonomously shuts down as the VCHP-W condenser closes upon WEB cooling. The VCHP/VCHP system is thus an autonomous thermal transport/switching system.

A quantitative assessment was performed to evaluate/characterize the LHP/VCHP and VCHP/VCHP systems in the following 15 design/operational categories: (1) switching concept; (2) ON conductance; (3) OFF conductance; (4) mass; (5) size/dimensions; (6) power; (7) operational limits; (8) heritage; (9) controllability/autonomy; (10) reliability/risk; (11) integration ease; (12) modularity; (13) testability; (14) scalability; and (15) thermal switchability. The assessment results are listed in Table 5. Based on those results, the LHP/VCHP was selected.

Table 5. Quantitative/Qualitative Assessment of LHP/VCHP and VCHP/VCHP Concepts

\begin{tabular}{|c|c|c|}
\hline Category & LHP/VCHP & VCHP/VCHP \\
\hline 1. Thermal Switching Concept & VCHP turns ON/OFF autonomously & "VCHP-W turns ON/OFF autonomously \\
\hline 2. ON Conductance & $12 \mathrm{~W} / \mathrm{K}$ & $7 \mathrm{~W} / \mathrm{K}$ \\
\hline 3. OFF Conductance & 0.007 W/K (VCHP only; lower if LHP included) & $0.007 \mathrm{~W} / \mathrm{K}$ \\
\hline 4. Mass & $3.1 \mathrm{~kg}$ (not including radiator blades) & $4.3 \mathrm{~kg}$ (not including radiator blades) \\
\hline 5. Size/Dimensions & 0.5 m length LHP evaporator & 0.5 m length VCHP-R evaporator \\
\hline 6. Power (Control) & $0 \mathrm{~W}$ (LHP* autonomously reacts to VCHP) & $0 \mathrm{~W}$ (VCHP-R** autonomously reacts to VCHP-W) \\
\hline 7. Operational Limits (Tilt) & $34^{\circ}$ (75\% of VCHP static height in $\left.1 / 6 g\right)$ & $16^{\circ}(75 \%$ of VCHP-R static height in $1 / 6 \mathrm{~g})$ \\
\hline 8. Heritage & 30 propylene LHPs in space (or in queue) & $>1000$ ammonia VCHPs in space \\
\hline 9. Controllability/Autonomy & system operates autonomously & system operates autonomously \\
\hline 10. Reliability/Risk & $>10$ yr life/low (breadboard LHP to be tested) & $>10$ yr life/low (all SS VCHP design) \\
\hline 11. Ease of Integration & LHP can use coiled (flexible) transport lines & VCHPs can use flexible bellows \\
\hline 12. Modularity & minimal (can enhance but will increase $\Delta \mathrm{T}$ ) & modest (can enhance but will increase $\Delta \mathrm{T}$ ) \\
\hline 13. Testability (Ground Testing) & maximum tilt $\sim 6^{\circ}$ in any axis & maximum tilt $\sim 3^{\circ}$ in any axis \\
\hline 14. Scalability & high (due to intrinsic LHP capabilities) & modest (can add additional VCHP-Rs) \\
\hline 15. Thermal Switching Details & $\mathrm{G}_{\mathrm{ON}} / \mathrm{G}_{\mathrm{OFF}}=1700$, rapid thermal switching & $\mathrm{G}_{\mathrm{ON}} / \mathrm{G}_{\mathrm{OFF}}=1100$, rapid thermal switching \\
\hline
\end{tabular}

* LHP to have polymer wick for low power startup, auto-shutdown; **VCHP-R freeze/thaw tolerant due to noncondensable gas (NCG) 


\section{PLANS}

To prove out the LHP/VCHP thermal management system concept for long duration lunar surface missions including ILN anchor nodes, NASA has recently funded ATK Space to design/build/test an LHP with a 0.25-0.50 m polymer wick evaporator with dual condensers (radiators) as shown in Figure 8. The design of the LHP will be based on miniaturized evaporators that were developed as part of the NASA ST-8 program (Bugby, 2005 and Ku, 2007). The objectives of this test program, which is Phase 1 of a two-phase effort (with each phase having a roughly 6-month duration), are as follows: (a) verify that the LHP will autonomously shut down when its evaporator heat load falls to a level consistent with a fully blocked VCHP, a condition that occurs when the lunar surface external environment cools, the WEB power is reduced, and the WEB temperature falls to near its minimum operational level; (b) verify that the LHP will autonomously start up when its evaporator heat load increases to a level consistent with a VCHP transitioning from blocked to unblocked, which occurs when the lunar environment warms, the WEB power is increased, and the WEB temperature rises above its minimum operational level; (c) verify that the LHP can function with multiple condensers (radiators), which may see different or identical lunar surface sink temperature environments; and (d) design the Phase 1 test system so that the additional features to be verified in Phase 2 of the program, such as a VCHP isothermalization/switching system and a WEB (simulator), can be easily incorporated into the Phase 2 test bed as shown in Figure 9. A future paper will describe the test plans/results.

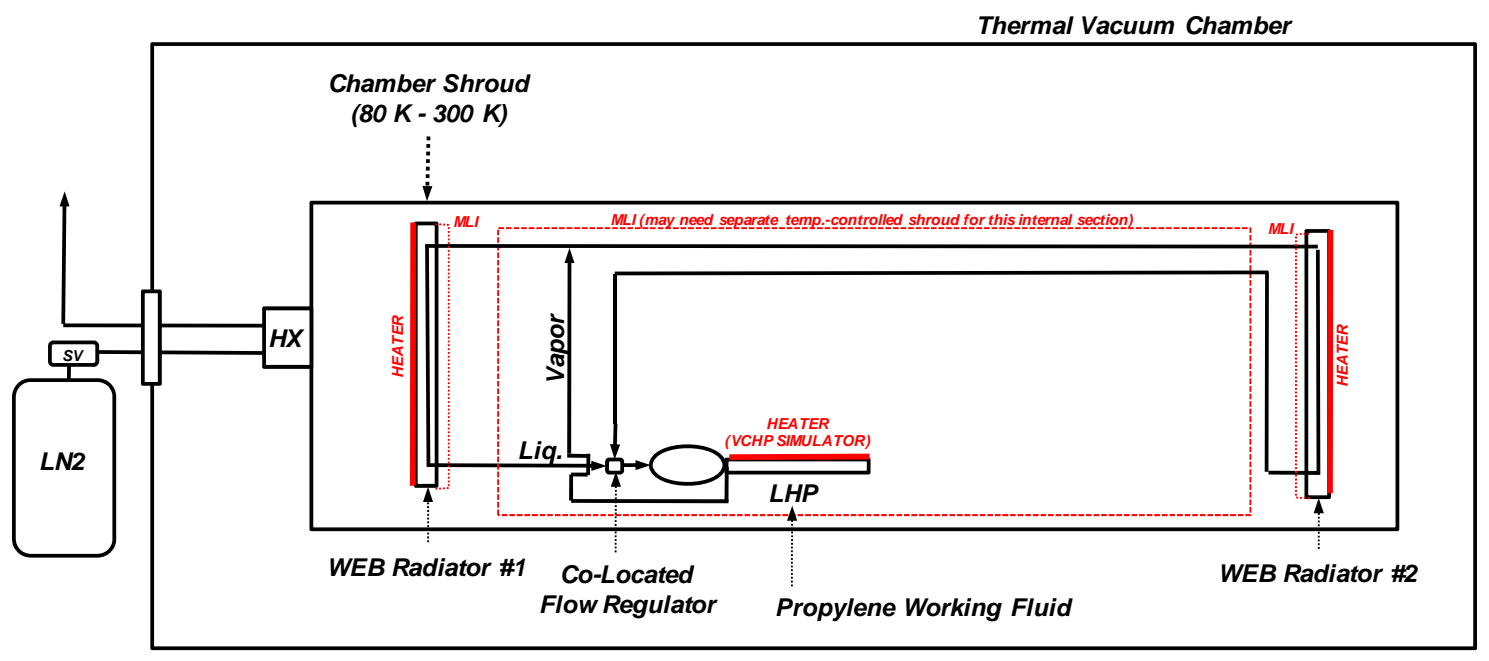

FIGURE 8. Phase 1 Test Set-Up

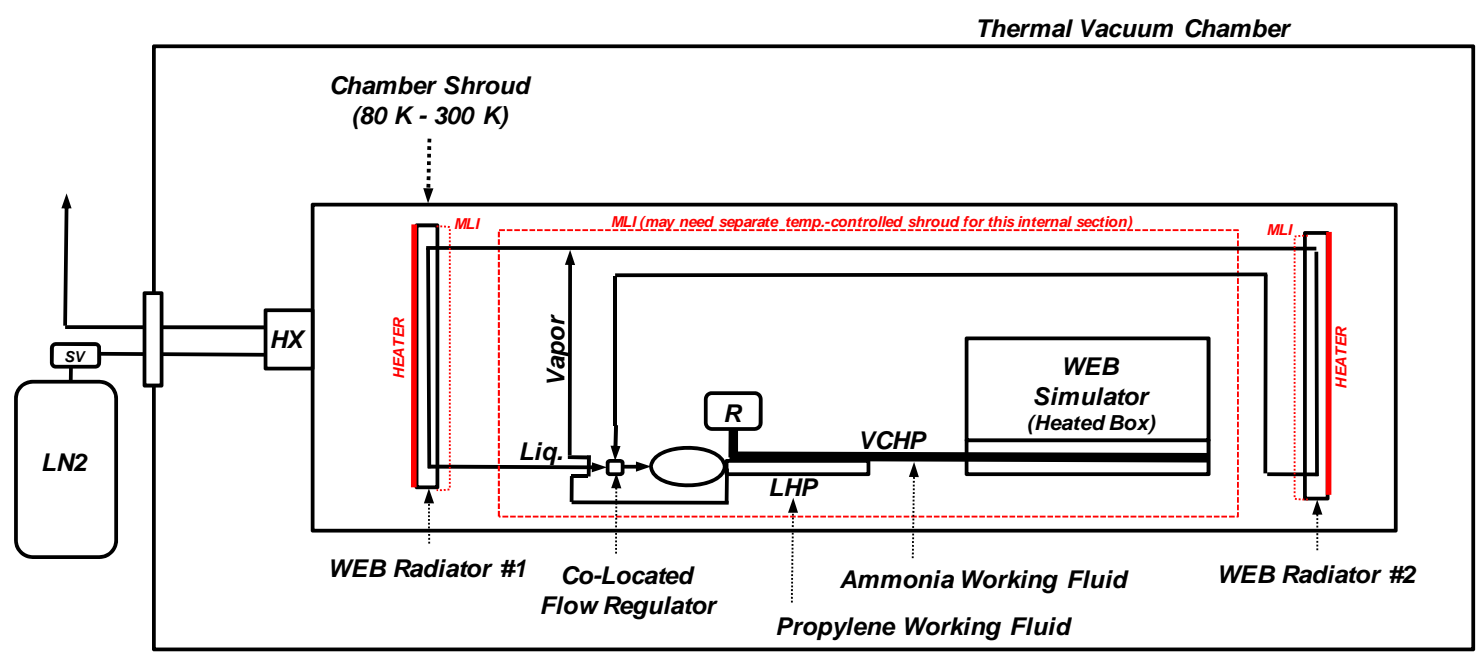

FIGURE 9. Phase 2 Test Set-Up 


\section{CONCLUSION}

The conclusion of the analytical study described herein is that the most appropriate thermal transport/switching system to meet the thermal management requirements of long duration lunar surface missions, the ILN anchor node mission in particular, is a cascaded system containing the following (two) subsystems: (1) a propylene $\mathbf{L H P}$ with a long, polymer wick evaporator (long for high conductance, polymer wick for low back conduction) that transports the requisite heat load to the radiator; and (2) dual ammonia VCHPs, the condensers for which interface to the LHP evaporator and the evaporators for which acquire heat from and isothermalize the WEB and its constituent electronic/battery elements. Thermal switching is provided by the VCHPs, the reservoirs for which are sized (with zero heater power) to turn VCHP transport OFF when the WEB temperature drops to near its minimum operating level, turn VCHP transport back ON when the WEB temperature begins to rise, and ensure the VCHP is fully ON when the WEB temperature approaches its maximum operating level. Of the 26 different system configurations that were analyzed, the LHP/VCHP system was found to best meet the six system discriminating requirements of low control power (none is required), long lifetime (a 10-year or more lifetime is expected), vertical integration (VI) capability (the system is able to isothermalize the WEB, perform the required thermal transport/switching, and isothermalize the radiator), low complexity (the system is based on straightforward flight-proven technologies), fully autonomous operation (the system operates without any power or active intervention), and tolerance to landed adverse tilt (the system is able to handle landed adverse tilts of about twice that required). As this paper was being written, a risk mitigation test program funded by NASA had just begun (at ATK Space) to design/build/test the LHP portion of the LHP/VCHP system. A future paper will describe this test program (Phase 1) and a possible follow-on test program (Phase 2) that may incorporate a VCHP and WEB simulator for a complete end-to-end demonstration of the LHP/VCHP thermal transport/switching system for future long duration lunar surface missions.

\section{ACRONYMS}

$\begin{array}{ll}\text { BV } & \text { - Bypass Valve } \\ \text { CCHP } & \text { - Constant Conductance Heat Pipe } \\ \text { CPL } & \text { - Capillary Pumped Loop } \\ \text { FV } & \text { - Flow-obstructing Valve } \\ \text { HLHP } & \text { - Hybrid Loop Heat Pipe } \\ \text { HX } & \text { - Heat Exchanger } \\ \text { HTS } & \text { - Heat Transport System } \\ \text { ILN } & \text { - International Lunar Network } \\ \text { LHP } & \text { - Loop Heat Pipe } \\ \text { MEC } & \text { - Multiple Evaporator/Condenser }\end{array}$

$\begin{array}{ll}\text { MP } & \text { - Multi-Pass } \\ \text { MPTPL } & \text { - Mechanically Pumped Two-Phase Loop } \\ \text { SC } & \text { - Subcooler } \\ \text { ST-8 } & \text { - Space Technology } 8 \\ \text { TEC } & \text { - Thermoelectric Cooler } \\ \text { VCHP } & \text { - Variable Conductance Heat Pipe } \\ \text { VCHTS } & \text { - Variable Conductance HTS } \\ \text { VI } & \text { - Vertical Integration } \\ \text { WEB } & \text { - Warm Electronics Box }\end{array}$

\section{ACKNOWLEDGMENTS}

The authors would like to gratefully acknowledge the funding support of the NASA Marshall Space Flight Center in the carrying out of this research.

\section{REFERENCES}

Bugby, D., Kroliczek, E., and Yun, J., “Development and Testing of a Miniaturized Multi-Evaporator Hybrid Loop Heat Pipe,” Space Technology and Applications International Forum (STAIF-2005), Albuquerque, NM (2005).

Ku, J., Ottenstein, L., Pham, T., and Douglas, D., “Miniature Loop Heat Pipe with Multiple Evaporators for Small Spacecraft

Thermal Control,” NASA Space Technology Conference (NSTC-2007), College Park, MD (2007). 